\title{
GOBIERNOS Y POLÍTICAS: TRANSFORMACIONES EN ARGENTINA Y AMÉRICA LATINA EN LA PRIMERA DÉCADA DEL SIGLO XXI
}

\section{GOVERNMENT AND POLICIES: CHANGES IN ARGENTINA AND LATIN AMERICA IN THE EARLY $21^{\text {ST }}$ CENTURY}

\section{Susana Hintze*}

\author{
RESUMEN
}

En años recientes comenzaron a gestarse en América Latina, procesos políticos nacionales en cuyo marco se observa la aparición de novedosas formas de organización, prácticas sociales y gobiernos que ponen en cuestión el orden neoliberal. En este trabajo se propone discutir las condiciones de surgimiento y las características de estos nuevos gobiernos, presentar sus políticas en términos de la cercanía/distancia con las del periodo neoliberal, así como, las transformaciones ocurridas y las tensiones que enfrentan.

PALABRAS CLAVE: NUEVOS GOBIERNOS * TRANSFORMACIONES POLÍTICAS * POLÍTICA SOCIAL * POLÍTICAS DE ECONOMÍA SOCIAL Y SOLIDARIA * TENSIONES POLÍTICAS

\section{ABSTRACT}

During recent years, innovative national political processes have developed in Latin American. These processes are characterized by the emergence of new forms of organization and for social practices and governments that challenge the neoliberal order. This article aims to discuss the emerging conditions and the characteristics of these new governments. Furthermore, it analyzes policies implemented by these governments in terms of similarity/dissimilarity with the ones implementing during neoliberal period, as well as, their changes and tensions.

KEYWORDS: NEW GOVERNMENTS * POLITICAL TRANSFORMATION * SOCIAL POLICY * SOCIAL AND SOLIDARITY ECONOMY POLICIES * POLITICAL TENSIONS

Universidad Nacional de General Sarmiento,

Argentina.

susana.hintze@gmail.com 


\section{INTRODUCCIÓN}

En un intento heterogéneo, que varía de país a país, en densidad y profundidad, en los aspectos abarcados y en los énfasis políticos, América Latina confronta el modelo que propugnó el neoliberalismo en el último tercio del siglo pasado. A comienzos del presente siglo, comenzaron a gestarse procesos políticos nacionales en cuyo marco se observa el surgimiento de novedosas formas de organización $y$ prácticas sociales que ponen en cuestión el orden neoliberal.

Una mirada sobre las últimas décadas muestra un fuerte contraste entre el pensamiento único y las políticas de ajuste estructural promovidas por el Consenso de Washington, que imperó de manera hegemónica, sobre todo en el decenio final del siglo pasado y en las propuestas de los gobiernos que han surgido en los primeros años de este siglo. El pasaje de Bucaram a Correa en Ecuador, de Sánchez de Losada a Evo Morales en Bolivia, de Menem a Kirchner en Argentina, solo para citar algunos personajes políticos; el tránsito de la sacralización del individuo y el mercado de la política de los 90 a los llamados a las democracias participativas y el "buen vivir" de las nuevas constituciones, son algunas pocas expresiones que ejemplifican las transformaciones acaecidas.

¿Cuán hondo han llegado estos cambios en la estructura económica y social de nuestros países? ¿Cuánto han modificado la existencia de sus habitantes en términos de condiciones de vida, pero sobre todo, igualdad y ampliación de derechos? Claramente menos de lo que auguran los nuevos discursos y a la vez muchísimo más de lo que era posible imaginar solo diez años atrás.

$1 \quad$ Inicialmente, en el gobierno de Evo Morales y luego en la Constitución Ecuatoriana de 2008, el "buen vivir" refiere no solo al "tener" sino sobre todo al "ser", "estar", "hacer" y "sentir". El buen vivir tiene que ver con el desarrollo en paz y armonía con la naturaleza; implica que las libertades, oportunidades, capacidades y potencialidades reales de los individuos se amplíen de modo que permitan lograr simultáneamente aquello que la sociedad, los territorios, las diversas identidades colectivas y cada uno valora como objetivo de vida deseable (Ramírez Gallegos, 2008).
No está de más señalar que las instituciones que están presentes actualmente, junto con los gobiernos y las políticas actuales no son entendibles sin la incorporación de la coyuntura en que se ubican. La consolidación de determinadas formas de integración internacional, de modelos de regulación, de mecanismos de representación e incorporación política están configurando los procesos macroeconómicos y políticos actuales, así como, la orientación, el alcance y el tipo de implementación de las políticas, en línea con la búsqueda de cambios progresivos dentro de las instituciones democráticas (Luna y Filgueira, 2009).

Del conjunto de los acontecimientos históricos que en una mirada de largo plazo, han contribuido a los procesos políticos en curso - bajo las diversas denominaciones de "progresistas", "nueva izquierda", "populistas" democráticos, radicales o redistributivos ${ }^{2}$ - no pueden excluirse las cortas y dramáticamente inconclusas experiencias de los 70. Dentro de las más relevantes se encuentra la de la Unidad Popular con Allende, en Chile, entre 1970-1973. Con un signo de tipo populista, se podría incorporar la que llevó a la brevísima "primavera camporista" en la Argentina en 1973³ los diez meses de gobierno del General Juan José Torres

2 Paramio (2006) los llama "populistas redistributivos", en contraposición al populismo liberal de un Menem o Fujimori en los 90. Una fuerte valoración del populismo se encuentra en Laclau (2005), en tanto, expresión de formas de liderazgo que no son ortodoxas desde el punto de vista liberal democrático y que aparecen cuando las masas populares que habían estado excluidas se incorporan a la arena política. A su criterio, el populismo, lejos de ser un obstáculo, garantiza la democracia, evitando que esta se convierta en mera administración.

3 Se conoce con este nombre a los poco menos de dos meses de gobierno de Héctor Cámpora, en cuya elección se cierra el periodo dictatorial 1966-1973. Apoyado por los sectores radicalizados del peronismo, renuncia luego del sangriento enfrentamiento que la derecha peronista produce durante la movilización popular, en ocasión de la llegada de Perón al país, en junio de 1973. Durante este corto lapso se liberan los presos políticos, se establecen relaciones con los gobiernos de Chile y Cuba, $y$ se intenta poner en marcha un pacto social entre capital y trabajo como sostén del modelo económico. 
en Bolivia (octubre 1970-agosto 1971, periodo en el cual destaca la nacionalización de minas y la expulsión del Cuerpo de Paz, organismo de cooperación norteamericano muy cuestionado por sus intervenciones en la región). Un periodo de más envergadura (1968-1975) lo constituye el proceso inicialmente encabezado por el General Velasco Alvarado, en Perú (nacionalización del petróleo, reforma agraria, industrialización, desarrollo de movilización popular vía el Sistema Nacional de Movilización Social/ SINAMOS).

Tomando en cuenta tales antecedentes, el trabajo se propone discutir las condiciones de surgimiento y las características de estos nuevos gobiernos en América Latina; presentar sus políticas en términos de la cercanía/distancia con las del periodo neoliberal, así como, las transformaciones ocurridas, con especial referencia a Argentina.

\section{CAMBIOS POLÍTICOS Y NUEVOS GOBIERNOS EN AMÉRICA LATINA A COMIENZOS DEL SIGLO XXI}

Las luchas que condujeron a los acontecimientos más arriba mencionados y la regresión política resultante de las dictaduras militares posteriores, han marcado la perspectiva histórica de toda una generación de políticos y militantes en América Latina y sobre la cual, se fueron construyendo estilos de liderazgos de muy distinto tipo, como el de Bachelet en Chile, Lula en Brasil o Néstor Kirchner en Argentina, para citar solo algunos ejemplos.

Si bien, es aún difícil predecir cuán profundas pueden ser algunas de las marcas sobre el cuerpo social de las nuevas tendencias en Latinoamérica y sobre todo, cuán reparadoras están siendo en las cicatrices de la etapa anterior, sin duda la hegemonía neoliberal está en declive $y$ entre sus grietas surgen espacios para nuevas formas de acción pública, que ponen en cuestión el proceso de ajuste, sus efectos en las condiciones concretas de vida de distintos sectores de la población, así como, las orientaciones que debe imprimirse a instituciones y políticas en esta etapa. Una muestra de ello, son las respuestas a la coyuntura derivada de la crisis mundial de 2008, que aún continúa enfrentada en la mayoría de nuestros países a través de políticas activas alejadas de las recetas del neoliberalismo.

En la literatura sobre las nuevas políticas, hay acuerdo con la descripción de la situación: buena parte de la población de América Latina vive hoy en el marco de gobiernos de nuevo tipo, si bien con características muy distintas. Se está incluyendo en este conjunto a los gobiernos de Argentina, Brasil, Bolivia, Ecuador, Nicaragua, Paraguay, Uruguay y Venezuela. También a Chile, hasta el cambio de Bachelet por Piñera y Honduras hasta el golpe institucional de 2009. Suele ser motivo de discusión si las categorías de "izquierda" y "derecha" tienen sentido en América Latina, en tanto, fueron inventadas en contextos parlamentarios europeos (Touraine, 2006). A pesar de su ambigüedad, el término "progresismo" es uno de los más generalizados para hacer referencia a los nuevos gobiernos y es el que se utilizará en este artículo para hacer genéricamente referencia al conjunto.

La privatización de la economía, la apertura de los mercados y la consiguiente desindustrialización de la etapa anterior han reconfigurado la región. Filgueira y otros (2010) consideran que no es simplemente la desigualdad, la pobreza y la exclusión resultante de dichos procesos lo que está en la base del desarrollo político de tales gobiernos. Son estas condiciones combinadas con los resultados de las transformaciones socieconómicas que experimentó la región en las últimas décadas del siglo pasado: la urbanización, la cual hace que cada vez más gente esté viviendo junta, convirtiéndose en trabajadores asalariados potenciales, aumentando la tasa de participación laboral en particular de las mujeres, en mercados de trabajo con poca capacidad de incorporarlos, fragmentados, caracterizados por la fuerte presencia de informalidad, precaridad y escasa protección, en los cuales se multiplican las iniciativas de trabajo por cuenta propia; el importante aumento de la escolarización (aunque esto hable más de la cantidad que de la calidad de la educación ofrecida); una inmersión en la "modernidad" debido a la exposición a nuevos patrones de consumo, con una fuerte brecha entre lo que ofrece la comunicación, la 
tecnología y los consumos a los que realmente puede acceder gran parte de la población. A todo ello, agregan un ingrediente político central, la experiencia de la democracia electoral durante los últimos 30 años: en contraste con la larga historia de periodos democráticos breves y extensos lapsos de dictaduras militares, entre 1979 y 1998, todos los países latinoamericanos pasaron a tener gobiernos elegidos democráticamente.

Señalan estos autores que la distancia entre la expansión de las aspiraciones al consumo y la movilidad social, frente al escaso dinamismo para democratizar la capacidad de satisfacer esas demandas en lo que hace a la estructura ocupacional, la redistribución del ingreso y riqueza y las garantías del bienestar, avalaron la percepción de una injusticia esencial que se expresó en movilizaciones de descontento contra las reformas neoliberales, en particular, en el momento de las crisis financieras de la región entre 1998-2002 (Luna y Filgueira, 2009; Filgueira y otros, 2010). De ella son herederos los nuevos gobiernos aquí considerados.

Algunos pocos datos permiten mostrar en cifras la situación de la región, la cual presenta una fuerte recuperación durante los primeros años del siglo. Entre 2003-2008 (cuando empieza a hacerse notar la recuperación post década de los 90 y antes de la crisis financiera global) la tasa de crecimiento del PIB en América Latina y el Caribe fue notablemente elevada y relativamente sostenida: en términos de crecimiento económico, es necesario retroceder hasta la década de 1970 para encontrar una fase de dinamismo económico similar ${ }^{4}$. La incidencia de la pobreza se redujo 11 puntos porcentuales (del $44 \%$ al 33\%) y la pobreza extrema disminuyó de alrededor del 19\% al $13 \%$, motivada por la reducción del desempleo, un incremento de los ingresos reales de la población ocupada, junto con la tendencia a la incorporación de las mujeres al mercado laboral y la reducción de la tasa de dependencia demográfica. En este mismo período, varios

$4 \quad$ La tasa anual de crecimiento fue del 2,6\% entre 1990 y 2002, se elevó al 4,9\% entre 2003 y 2008 (CEPAL, 2010). países latinoamericanos registraron una mejora en la distribución del ingreso: en 10 de 20 países el índice de concentración de Gini se redujo entre el $3 \%$ y el 10\%, con la excepción de Venezuela en donde la reducción de la desigualdad medida por este indicador fue mayor (18\%). Sin embargo, es preciso relativizar este progreso:

.. ya que la mitad de los países de la región no lograron un avance significativo en materia de desigualdad y solo tres de los sietes países de menor desarrollo relativo registraron una disminución del índice de Gini. América Latina y el Caribe sigue siendo la región con peor distribución del ingreso del mundo (CEPAL, 2010: 1).

En lo que hace a las razones geopolíticas y políticas que moldean la génesis y las características de estos gobiernos, se encuentra la ruptura del mundo bipolar, que condensa simbólicamente la caída del Muro de Berlín (Natanson, 2008). Según el autor citado, esto los aleja del modelo de izquierda revolucionaria clásica del siglo XX y los hace compartir una visión pragmática, orientada a resolver los problemas nacionales del presente con fórmulas flexibles y mirados más en términos de inclusión y desarrollo que de revolución ${ }^{5}$. La excepción es Venezuela, donde se caracteriza así al proceso; o el apelativo de "revolución ciudadana" que utiliza Correa en Ecuador. Llegan al gobierno nacional por una variedad de caminos, que en algunos de los casos son resultado de un proceso gradual; a partir de los gobiernos estaduales y municipales construyen ejemplos de lo que es

$5 \quad$ El filósofo argentino Carlos Altamirano coincide con esta apreciación. Considera al kirchnerismo como "un movimiento de reformas sociales, centrado sobre todo en una nueva distribución. A los ojos de un setentista de 1972 o 1973; sin embargo, es un proyecto redistribucionista, no uno que apunte, hasta ahora, a cambiar las estructuras sociales. Junto al reformismo social, está la incorporación de derechos de nuevo tipo, como el relativo al matrimonio igualitario" (Entrevista con Carlos Altamirano. "El kirchnerismo es el hecho maldito del progresismo". Diario La Nación, Buenos Aires 18/09/2011). 
posible hacer cuando arriben a nivel nacional (el PT en Brasil y el Frente Amplio en Uruguay son los casos de mayor peso), caminos que en general, recorren junto con un nuevo conjunto de protagonistas. Si la izquierda clásica asumía como sujeto histórico al proletariado industrial, no obstante, las condiciones creadas por el capitalismo financiero globalizado que lo limitó numéricamente y debilitó su organización, les han mostrado la importancia del reconocimiento de la sociedad civil y la diversidad, el avanzar junto con los movimientos $y$ organizaciones sociales, culturales, ecologistas, grupos étnicos, de género, de lucha por el reconocimiento de la identidad sexual, entre otros. Finalmente, los aúna el origen democrático: todos los gobernantes han asumido como resultado de procesos electorales, en algunos casos revalidados varias veces $y$ en otros, empujando sus límites en lo que hace a la duración de los mandatos (Natanson, 2008). Todos reconocen la importancia de preservarla, como lo demuestran las rápidas y unánimes respuestas políticas frente a los intentos de romper la legalidad democrática en Bolivia, Honduras y Ecuador en los últimos años, en las cuales tuvó un importante papel la UNASUR (Unión de Naciones Suramericanas constituida en 2008). Expresión a su vez, de otra de las características de estos procesos: la valoración de la identidad y unidad continental y un nuevo posicionamiento frente al norte.

Juntos con estos aspectos comunes, los gobiernos considerados difieren mucho entre sí y las clasificaciones de orden político pueden resultar simplificadoras. Así se les agrupa en gobiernos populistas de nuevo tipo, surgidos en países en que virtualmente se fragmentó el sistema de partidos histórico (Bolivia, Ecuador, Venezuela y Paraguay) y en gobiernos con un perfil más socialdemócrata (Chile hasta Bachelet, Uruguay y Brasil) con partidos políticos consolidados que los apoyan. Argentina es un caso particular, en el cual el gobierno proviene de un antiguo partido nacional popular cuyos postulados históricos fueron revertidos por un gobierno del mismo signo en los 90, el menemismo (Gudynas y otros, 2008; Filgueira y otros, 2010; Lanzaro, 2007).
Otro tipo de clasificación pone el acento en los cambios institucionales que han promovido y en este caso, se observan dos grandes tendencias: la de los gobiernos que propugnan una refundación institucional de base en la forma de reformas constitucionales (Bolivia, Ecuador y Venezuela); un segundo grupo, el de los restantes países, en los cuales los nuevos gobiernos se insertaron en el orden político vigente. Argentina es nuevamente un caso atípico: viniendo de un profundo cuestionamiento de los políticos en el 2001, ha reconstituido una representación política (en particular parlamentaria) en la que proliferan aquellos a los cuales se les dedicaba la frase "que se vayan todos" (Gudynas y otros, 2008). En este marco, sin embargo, en Argentina se han realizado transformaciones que impulsan una nueva institucionalidad, entre los más relevantes, el cambio de la Corte Suprema de Justicia, la derogación de las leyes de impunidad que impedían juzgar a los culpables de delitos de lesa humanidad cometidos durante la última dictadura militar, la ley de medios audiovisuales que regula los servicios de comunicación vía mecanismos destinados a la desconcentración y fomento de la competencia de las nuevas tecnologías de información y comunicación, y la contra reforma provisional con la re-estatización del sistema de jubilaciones y pensiones.

Este conjunto de gobiernos está implementando muy diversas $y$ heterodoxas intervenciones, sin embargo, coinciden todos en una fuerte valoración del papel del estado y su capacidad para generar políticas activas. En su análisis del "giro a la izquierda" en América Latina, Ludolfo Paramio lo expresa de esta manera:

... dentro de los gobiernos que podemos considerar de izquierda existe una llamativa divergencia en el discurso político, en la postura respecto al proceso de globalización y en la interpretación de las instituciones democráticas y sus reglas de juego. La única coincidencia explícita es el hincapié en la política social y en la búsqueda de un modelo económico que no solo produzca crecimiento, sino también resultados sociales: creación 
de empleo, mejora de la educación y la salud, reducción de la pobreza y la indigencia (Paramio, 2006: 64).

\section{SOBRE LAS POLÍTICAS IMPLEMENTADAS: LO VIEJO Y LO NUEVO}

En lo que hace a las políticas llevadas a cabo por estos nuevos gobiernos, una cuestión que requiere de investigaciones específicas es la de la continuidad/ruptura entre el discurso antineoliberal explícito en estos gobiernos y las intervenciones que han desarrollado. A pesar de los incrementos en el gasto social, los cambios en su masividad o el aumento en los beneficios, parte de los programas sociales siguen teniendo características del periodo anterior, son focalizados y mantienen las contraprestaciones.

En ese plano es interesante el análisis de Luna y Filgueira (2009), respecto a las herramientas de política puestas en práctica por los nuevos gobiernos de la región según el tipo de orientaciones políticas que presentan. Entre ellas se encuentran:

a) Herramientas de ascendencia neoliberal: los programas de transferencia condicionada de ingresos, establecidos en los 80 y 90 en casi todos los países latinoamericanos, se mantienen y en algunos casos, son eje de la política social como el Bolsa Familia, en Brasil ${ }^{6}$. Otros ejemplos son

$6 \quad$ Bolsa Familia es uno de los núcleos centrales del programa Hambre Cero (Fome Zero), estrategia del gobierno Lula para impulsar el derecho a la alimentación de la población vulnerable. Con más de 11 millones de familias beneficiadas $(24 \%$ de la población) ha sido central en la caída de la tasa de indigencia que pasó del 14\% al 7\% entre 20022010. Es un programa de transferencia condicionada de ingreso, relacionado también a acciones de economía solidaria. Coexistió con su antecesor, el programa Comunidad Solidaria del gobierno de Fernando Henrique Cardozo, hasta que fueron unificados todos los programas de transferencia de ingresos en 2004; desde entonces tendió a la universalización de la cobertura de todas las personas en situación de extrema pobreza y se amplió el nivel de ingreso familiar que define el acceso al beneficio. A partir de 2007, se agregó la recomposición del valor de los beneficios y el aumento de
Chile Solidario, Bono de Desarrollo Humano en Ecuador, Hambre Cero en Nicaragua y el Programa Familias en Argentina.

Los cuasi mercados en educación, salud y seguridad social fueron también un recurso de la estrategia neoliberal mantenido en algunos países, siendo el caso prototípico el de la Concertación en Chile (recién con Bachelet se inició una limitada reforma del sistema de jubilaciones $y$ pensiones privadas). Los autores mencionados encuentran la razón de la perduración de estas herramientas, entre otras, en la conveniencia política (alta legitimidad respecto del costo), la inercia institucional y la limitación de recursos.

b) Herramientas de tradición socialdemócrata: absteniéndose de compararlas con las europeas, las ubican en este campo, en tanto, estrategia de gradual construcción de ciudadanía a través de una regulación moderada de los mercados y énfasis en la equidad. Incluyen entre estas herramientas a los programas que se proponen universalistas, entre ellos: el Plan Equidad en Uruguay (2007); el Régimen de Garantías Explícitas en Salud, en Chile, durante el gobierno de Lagos que garantiza acceso universal y el Programa Chile Crece Contigo impulsado por Bachelet, consistente en un sistema de protección social integral de la infancia, desde el embarazo hasta los cuatro años. Se les puede agregar la Asignación Universal por Hijo para Protección Social, en Argentina (2009), que extiende un beneficio que hasta el momento percibían los trabajadores en relación de dependencia a los informales $y$ desocupados.

cobertura, con la inclusión de los jóvenes de 16 a 17 años. Para estimar el efecto del programa Bolsa Familia puede señalarse que el total del beneficio al que puede acceder una familia en extrema pobreza alcanzaba en 2010 a unos US\$ 100, siendo alrededor de US\$ 300 en Brasil, el nivel de ingresos del grupo de ingresos bajos. En: <www.mds.gov. br/bolsafamilia/beneficios/composicao-de-valores $>$ [consultado 10 de diciembre de 2010]. 
Las reformas tributarias/impositivas, recurso típico de una estrategia socialdemócrata, no han sido encaradas por los gobiernos progresistas, a pesar del reconocimiento de su necesidad. Requieren de alianzas sociales sólidas que por el momento no se han concretado.

c) Herramientas populistas radicales: se incluyen en este apartado campañas sociales (entre las más significativas: las "misiones" de Venezuela7; si bien, hay experiencias similares como las

$7 \quad$ Las "misiones" son una nueva forma de organizar la gestión de las políticas sociales gestada por el gobierno Chávez a partir de 2003, luego del fracasado intento de destitución del presidente (abril 2002) y de la derrota del paro petrolero apoyado por un boicot empresarial. Originalmente, fueron la respuesta gubernamental ante las dificultades encontradas para reorientar la gestión pública, a través de las organizaciones administrativas estatales existentes, caracterizadas por la ineficiencia, clientelismo y corrupción, así como, por la resistencia por parte de la burocracia estatal a muchas de sus nuevas políticas, debido a la oposición de sindicatos de la administración pública con hegemonía de los partidos tradicionales. En la práctica constituyen la reorientación de las políticas sociales focalizadas que se habían generalizado en todo el continente hacia políticas sociales universalistas, con búsqueda de la participación popular (Lander, 2007). Se gestionan por fuera de las estructuras de línea de los ministerios y cuentan con recursos extraordinarios, bajo coordinación interinstitucional e interministerial (En: $<w w w$. pdvsa.com>, 2008). La inicial fue la Misión Barrio Adentro, destinada a la atención de la salud, con el apoyo de personal médico cubano, le siguieron las educativas (Misión Robinson) y luego, se establecieron misiones para cada una de las áreas de las políticas sociales o para intervenciones específicas. En relación con la economía social, destacan la Misión Vuelvan Caras (capacitación para el trabajo y generación de empleo), la de entrega de tierra y apoyo a los campesinos (Misión Zamora), el sistema nacional público de distribución de alimentos a bajo precio (Misión Mercal), la atención directa de los sectores más excluidos (Misión Negra Hipólita), los pueblos indígenas (Misión Guaicaipuro), la atención a madres más pobres (Misión Madres del Barrio) y la de vivienda y redistribución territorial (Misión Villanueva). A ellas se suman muchas otras (ver Gobierno en Línea. En: <www.gobiernoenlinea.ve/miscelaneas/misiones.html> [consultado el 10 de diciembre de 2010]). campañas de alfabetización y salud en Bolivia, Ecuador y Nicaragua. Dentro de este grupo de instrumentos incorporan también, los impuestos a las exportaciones estratégicas (retenciones a las exportaciones agropecuarias en Argentina, a los hidrocarburos en Bolivia, petróleo en Ecuador), subsidios a determinados sectores, controles de precios, incrementos de salarios y nacionalización de empresas, en particular, en Venezuela (Luna y Filgueira, 2009).

En lo específico a las políticas sociales, un aspecto a tomar en cuenta de acuerdo con lo ocurrido en esta primera década del siglo, refiere a la cuestión de la gestión. Las falencias en la eficacia $y$ cobertura de los programas sociales siguen presentes en muchos países. El clientelismo y la corrupción resultan muy difíciles de superar, a pesar de los discursos que los cuestionan. Acerca de la participación - tema también muy presente en los discursos de estos gobiernos- es mucho lo que hay todavía por hacer en una concepción de la participación que trascienda la instrumental que propició el neoliberalismo.

\section{LA ECONOMÍA SOCIAL Y SOLIDARIA Y SUS POLÍTICAS EN AMÉRICA LATINA}

A las herramientas de política reseñadas - y no fácilmente encasillables en la tipología anterior que responde a una visión clásica de la política- se les suman otro tipo de intervenciones, las de economía social y solidaria, que aparecen en la agenda de la mayoría de gobiernos mencionados anteriormente y que han implicado iniciativas de respuesta a la cuestión del trabajo y la reproducción, vía el trabajo asociativo y autogestionado con propiedad colectiva o social.

Tales políticas son denominadas de distinta manera y ello responde a diferencias conceptuales, ideológicas y valorativas, e incluso de procedencia geográfica, referidas a un campo en construcción que en nuestra región comienza a tomar mayor peso a fines de la década pasada. Sus contenidos están aún definiéndose 
y comienzan a expresarse en novedosas formas institucionales ${ }^{8}$.

En los gobiernos que desarrollaron una refundación institucional, la economía social y solidaria aparece en las nuevas constituciones ${ }^{9}$.

Así, la Constitución de la República Bolivariana de Venezuela, promulgada en 1999, en el artículo 184 establece que "la ley creará mecanismos abiertos y flexibles para que los Estados y los Municipios descentralicen y transfieran a las comunidades y grupos vecinales organizados los servicios que éstos gestionen previa demostración de su capacidad para prestarlos". Para ello se propone promover: "la participación en los procesos económicos estimulando las expresiones de la economía social, tales como cooperativas, cajas de ahorro, mutuales y otras formas asociativas". A su vez, el artículo 308 declara que:

8 En un intercambio de una lista sobre economías alternativas, levantado por la revista Otra Economía, un grupo de destacados intelectuales latinoamericanos expuso su interpretación acerca de la mejor manera de denominar a las experiencias y movimientos inspirados por valores solidarios y articulados alrededor del trabajo asociativo. El espectro incluyó "socieconomía solidaria" (que refiere al concepto de economía imbricada de K. Polanyi y entronca con el movimiento de socioeconomía) y entre los que consideran pertinente seguir utilizando economía, el de economía solidaria, economía de solidaridad, economía social, economía del trabajo (Guerra, 2007). La denominación de "economía social y solidaria" se ha ido constituyendo en el nombre genérico, que reconociendo estas diferencias, provisoriamente las salva al unificar las dos más extendidas. Es la utilizada por las redes como RIPESS (Red Intercontinental de Promoción de la Economía Social y Solidaria) y RILESS (Red de Investigadores en Economía Social y Solidaria).

La economía social, en particular con referencia a las cooperativas, ha estado presente en algunas constituciones latinoamericanas. Por ejemplo, en Venezuela, el sector cooperativo tiene rango constitucional desde 1961; en Ecuador, ya la Constitución de 1978 considera que "la economía ecuatoriana opera y se desenvuelve con la concurrencia y la coexistencia de los sectores: público, privado, mixto; y comunitario de autogestión" (art. 61). Lo novedoso en todo caso es el desarrollo y peso que esta concepción toma en las nuevas constituciones y como se va configurando en la normativa y prácticas posteriores (ver Hintze, 2010).
El Estado protegerá y promoverá la pequeña y mediana industria, las cooperativas, las cajas de ahorro, así como también la empresa familiar, la microempresa y cualquier otra forma de asociación comunitaria para el trabajo, el ahorro y el consumo, bajo régimen de propiedad colectiva, con el fin de fortalecer el desarrollo económico del país, sustentándolo en la iniciativa popular. Se asegurará la capacitación, la asistencia técnica y el financiamiento ${ }^{10}$.

La Constitución boliviana de 2008, en el capítulo referido a la organización económica del Estado, establece que "el modelo económico boliviano es plural y está orientado a mejorar la calidad de vida $y$ el vivir bien de todas las bolivianas y los bolivianos". Define los contenidos de "la economía plural", conformada "por las formas de organización económica comunitaria, estatal, privada y social cooperativa"; así como, los principios $y$ valores en que se sustenta:

La economía plural articula las diferentes formas de organización económica sobre los principios de complementariedad, reciprocidad, solidaridad, redistribución, igualdad, seguridad jurídica, sustentabilidad, equilibrio, justicia y transparencia. La economía social y comunitaria complementará el interés individual con el vivir bien colectivo (artículo 306) ${ }^{11}$.

10 Constitución de la República Bolivariana de Venezuela. En: <www.constitucion.ve>. La manera de entender esta forma de organización económica fue modificándose (incluso en la denominación) en los Planes de Desarrollo Económico y Social de 2001 y 2007, así como, en la normativa específica.

11 En otros artículos, se establece el reconocimiento, el respeto y la protección estatal de la organización económica comunitaria ("sistemas de producción y reproducción de la vida social, fundados en los principios $y$ visión propios de las naciones y pueblos indígena originario y campesinos", artículo 307); de "las cooperativas como formas de trabajo solidario y de cooperación, sin fines de lucro" y la promoción prioritaria de la organización de cooperativas en actividades de producción (artículo 310). A su vez, define que "todas las formas de organización 
La nueva Constitución de Ecuador, también de 2008, define al "sistema económico como social y solidario", entendiendo que "propende a una relación dinámica y equilibrada entre sociedad, Estado y mercado, en armonía con la naturaleza; $y$ tiene por objetivo garantizar la producción y reproducción de las condiciones materiales e inmateriales que posibiliten el buen vivir". Reconoce como integrante del sistema económico a "las formas de organización económica pública, privada, mixta, popular y solidaria". En lo que hace a la "economía popular y solidaria", su composición abarca "los sectores cooperativistas, asociativos y comunitarios" (artículo 283) ${ }^{12}$.

En general, si bien con distintas perspectivas, en la mayoría de países de América Latina se están llevando a cabo políticas de promoción de experiencias de este tipo. De manera simplificada $-y$ reconociendo los riesgos de las clasificaciones polares- estas intervenciones en algunos casos no van más allá de acciones de promoción del autoempleo como medio para la autosustentación, básicamente, a través de microemprendimientos. Constituyen una forma más de apoyo a sectores vulnerables excluidos del mercado de trabajo (formal o informal), en el marco de las políticas sociales asistenciales, generalmente, con escasos recursos presupuestarios y de gestión. En el otro extremo se encuentran los enfoques que plantean la posibilidad de conformarla en una estrategia que dispute espacios con el capitalismo o que se convierta a largo plazo, en un alternativa superadora de este.

económica establecidas en esta Constitución gozarán de igualdad jurídica ante la ley" (artículo 311). Similar reconocimiento a la economía plural se encuentra en los artículos constitucionales referidos a políticas económicas, financieras, sectoriales $y$ de desarrollo rural integral sustentable (Nueva Constitución Política del Estado. En: <www.presidencia.gob.bo/download/constitucion.pdf $>$ [consultado el 6 de setiembre de 2011]).

12 Constitución del Ecuador. En: <www.asambleanacional.gov.ec/documentos/constitucion_de_bolsillo.pdf.> [consultado el 6 de setiembre de 2011].

\section{A MODO DE CIERRE: CUESTIONES QUE PONEN EN TENSIÓN LOS NUEVOS ESCENARIOS Y SU CONTINUIDAD}

Para concluir cabe señalar que, en los últimos años, algunas cuestiones han puesto en tensión los escenarios políticos en la región y su perdurabilidad en el tiempo: por un lado la crisis global del capitalismo; si bien, América Latina hasta ahora parece estar cursándola con bastante éxito, la situación en los países del capitalismo avanzado crea espacios de incertidumbre.

Para la CEPAL, un hecho que marca una diferencia con las crisis anteriores en América Latina, es que en esta oportunidad no han sido los problemas financieros los centrales, gracias a que los países están menos endeudados y tienen más reservas internacionales, a la vez que los sistemas financieros regionales presentan un grado de exposición externa relativamente bajo. En la última parte de 2008 y en los primeros meses de 2009, los impactos más fuertes provinieron de la economía real, relacionados con una significativa disminución de los flujos de comercio internacional, un deterioro de los términos de intercambio y la disminución de las remesas, elementos impulsores del crecimiento regional en los años precedentes (CEPAL, Comunicado de Prensa, 10 de junio de 2009. En: www.cepal.org). Las políticas activas puestas en marcha por la mayoría de los países operaron de manera anticíclica moderando, hasta el momento, los efectos más adversos.

Por el otro lado, en lo interno, cabe destacar el resurgimiento de nuevo cuño de las rupturas democráticas que han tomado una progresión importarte desde el intento fracasado en Venezuela, en el 2002; la asonada separatista en Bolivia, en el 2008; el derrocamiento del presidente Manuel Zelaya en Honduras, en 2009 y el atentado en Ecuador contra el presidente Correa en setiembre de 2010. Junto a ello, la asunción de Dilma Rousseff, en Brasil muestra la continuidad del rumbo.

A pesar de los avances y las limitaciones de los procesos políticos de la presente década, no hay dudas de que esta es otra América Latina con preocupaciones, intereses y objetivos sociales muy diferentes a los del periodo neoliberal: diferente en tanto aspiración de los movimientos y sectores populares, de los actores 
políticos que lideran los procesos y en tanto, discurso académico/técnico predominante ${ }^{13}$. En palabras de Laclau: "en los últimos 20 años, por primera vez en la historia latinoamericana, las aspiraciones nacionales y populares de las masas logran coincidir con la afirmación de los derechos humanos, la división de poderes, el pluralismo político"14.

\section{BIBLIOGRAFÍA}

\section{LIBROS}

Filgueira, Fernando; Reygadas, Luis; Luna, Juan Pablo y Alegre, Pablo. "Shallow states, deep inequalities and the limits of conservative modernization: the politics and policies of incorporation in Latin America". The great gap: the politics of inequality in Latin America. Blofield, Merike. Penn State University Press, 2010.

Gudynas, Eduardo; Guevara, Rubén y Roque, Francisco (coord.). Heterodoxos. Tensiones y posibilidades de las políticas sociales en los gobiernos progresistas de América del Sur. Montevideo: Centro Latinoamericano de Ecología Social/ CLAES, 2008.

Hintze, S. La política es un arma cargada de futuro. La economía social y solidaria en Brasil y Venezuela. Buenos Aires: CLACSO-CICCUS, 2010.

Laclau, Ernesto. La razón populista. Buenos Aires: FCE, 2005.

Natanson, José. La nueva izquierda. Triunfos y derrotas de los gobiernos de Argentina, Brasil, Bolivia, Venezuela, Chile, Uruguay y Ecuador. Buenos Aires: Debate, 2008.

Ramírez Gallegos, Rafael. Igualmente pobres, desigualmente ricos. Quito: Ariel, 2008.

13 Aunque siga pendiente la pregunta de sino se está en presencia de un nuevo movimiento del péndulo anti-establishment, al cual la historia latinoamericana es proclive (Luna y Filgueira, 2009 y Natanson, 2008).

14 Ernesto Laclau: "El populismo garantiza la democracia”. entrevista en diario La Nación, Buenos Aires. Fecha: 10 de mayo de 2005.

\section{REVISTAS}

Lander, Edgardo. "El Estado y las tensiones de la participación popular en Venezuela". OSAL VIII (22). Buenos Aires. ClACSO, 2007: 65-85.

Lanzaro, Jorge. "Gobiernos de izquierda en América Latina: entre el populismo y la social democracia. Una tipología para avanzar en el análisis comparado". Análise de Conjuntura 12. IUPERJ, 2007: $1-20$.

Luna, Juan Pablo y Filgueira, Fernando. "The left turns as multiple paradigmatic crises". Third World Quarterly 30 (2). Routledge. Marzo, 2009: 371-395.

Paramio, Ludolfo. "Giro a la izquierda y regreso al populismo". Revista Nueva Sociedad 205. Friedrich Ebert Stiftung. Setiembre-octubre, 2006: 62-74.

Touraine, Alain. "Entre Bachelet y Morales ¿existe una izquierda en América Latina?" Revista Nueva Sociedad 205. Friedrich Ebert Stiftung. Setiembreoctubre, 2006: 46-55.

\section{TEXTOS ELECTRÓNICOS}

CEPAL. El progreso de América Latina y el Caribe hacia los Objetivos de Desarrollo del Milenio. Desafíos para lograrlos con igualdad. Naciones Unidas, 2010. En: < http://www.eclac.org/cgi-bin/getProd. asp? xml=/publicaciones/xml/1/39991/ P39991.xml\&xsl=/tpl/p9f.xsl\&base $=/$ tpl/top-bottom.xsl> [consultado $6 \mathrm{de}$ setiembre de 2011].

Guerra, Pablo (org.). "Cómo denominar a las experiencias económicas solidarias basadas en el trabajo. Diálogo entre académicos latinoamericanos acerca de la polémica conceptual". Otra Economía I (1). 2do. Semestre, 2007: 21-27. En: $<$ http://revistaotraeconomia.blogspot. com/2007/10/cmo-denominar-lasexperiencias_06.html> [consultado 6 de setiembre de 2011].

Fecha de ingreso: 29/11/2011

Fecha de aprobación: 10/02/2012 